\title{
A characterization of efficient points in constrained location problems with regional demand
}

\author{
Emilio Carrizosa ${ }^{\mathrm{a}, *}$, Frank Plastria $^{\mathrm{b}}$ \\ a Facultad de Matemáticas, C/Tarfia s/n, 41012 Sevilla, Spain \\ ${ }^{\mathrm{b}}$ Center for Industrial Location and Development, Pleinlaan 2, B 1050 Brussels, Belgium \\ Received I January 1995; revised I February 1996
}

\begin{abstract}
In this paper we characterize the set of efficient points in the planar point-objective location problem under a convex locational constraint, when distances are measured by a strictly convex norm in $\mathbb{R}^{2}$ and the set of demand points is a compact set.

It is shown that, under these assumptions, the efficient set coincides with the closest-point projection of the convex hull of the demand points onto the feasible set.
\end{abstract}

Keywords: Location problems; Efficiency; Convex analysis; Strictly convex norms

\section{Introduction}

Let $A$ and $S$ be nonempty sets in $\mathbb{R}^{2}$, and let $\gamma$ be a norm in $\mathbb{R}^{2}$. Consider the vector-optimization problem $\mathbf{P}(\gamma, A, S)$,

$\mathbf{P}(\gamma, A, S): \quad \min _{x \in S}\left(\gamma_{a}(x): a \in A\right)$,

where, for each $a \in A, \gamma_{a}$ is the function

$\gamma_{a}: x \in \mathbb{R}^{2} \rightarrow \gamma_{a}(x)=\gamma(x-a)$

measuring the distance up to $a$.

A point $x \in S$ is said to be efficient for problem $\mathbf{P}(\gamma, A, S)$ iff there exists no $y \in S$ such that $\gamma_{a}(y) \leqslant \gamma_{a}(x)$ for all $a \in A$, with at least one strict inequality. A point $x \in S$ is weakly efficient iff there exists no $y \in S$ such that $\gamma_{a}(y)<\gamma_{a}(x)$ for all $a \in A$.

* Corresponding author.
Throughout this note, the set of efficient and weakly efficient points for $\mathbf{P}(\gamma, A, S)$ will be denoted, respectively, $\mathbf{E}(\gamma, A, S)$ and $\mathbf{W E}(\gamma, A, S)$.

A number of papers (see e.g. $[2,4,6,10,14,15]$ ) have been devoted to the search of efficient points of the problem above, known in the literature as the pointobjective location problem (see [13]), but mostly in the unconstrained case, i.e., under the assumption that the facility can be placed at any point in the plane, i.e., $S=\mathbb{R}^{2}$.

Although this assumption has been widely questioned (see, e.g., [5]), only some partial results have been obtained in the presence of constraints. For instance, in $[5,9]$ necessary conditions for a point to be efficient are derived, e.g., the points in $\mathbf{E}(\gamma, A, S)$ are visible from the set $\mathbf{E}\left(\gamma, A, \mathbb{R}^{2}\right)$ of efficient points for the unconstrained problem [9]. However, a full characterization of the set of (weakly) efficient points has 
only been obtained in [1] for the classical case when $\gamma$ is the euclidean norm and $A$ is finite, showing that $\mathbf{E}(\gamma, A, S)$ and $\mathbf{W E}(\gamma, A, S)$ coincide with the orthogonal projection onto $S$ of $\mathbf{E}\left(\gamma, A, \mathbb{R}^{2}\right)$, known to equal the convex hull of $A$ [13].

In this paper we characterize $\mathbf{E}(\gamma, A, S)$ and WE $(\gamma, A, S)$ when $A$ is compact, $S$ is a closed convex set and $\gamma$ is a strictly convex norm, (i.e., $\gamma$ is a norm such that the boundary of its unit ball does not contain nondegenerate line segments), showing that both $\mathbf{E}(\gamma, A, S)$ and $\mathbf{W E}(\gamma, A, S)$ equal the closest-point projection (with respect to $\gamma$ ) of the convex hull of $A$ (i.e., $\left.\mathbf{E}\left(\gamma, A, \mathbb{R}^{2}\right)=\mathbf{W E}\left(\gamma, A, \mathbb{R}^{2}\right)[13]\right)$.

Since the euclidean norm is strictly convex, the characterization given in [1] is extended here in two ways: $A$ is allowed to be infinite, and $\gamma$ is an arbitrary strictly convex norm.

The proofs make use of rather well-known results of Convex Analysis, which may be found, e.g., in [12].

\section{The results}

In what follows, $S$ is a nonempty closed and convex set in $\mathbb{R}^{2}, A$ is a nonempty compact subset of $\mathbb{R}^{2}$, and $\gamma$ is a strictly convex norm, whose unit ball is denoted by $B$; the dual norm of $\gamma$ is denoted by $\gamma^{0}$, and its unit ball by $B^{0}$.

Given a set $X \subset \mathbb{R}^{2}$, let $\operatorname{conv}(X)$ denote its convex hull, and $b d(X)$ its boundary; for any $x \in X$, let $X^{*}(x)$ be the convex cone

$X^{*}(x)=\left\{u \in \mathbb{R}^{2}:\langle u, y-x\rangle \geqslant 0\right.$ for all $\left.y \in X\right\}$,

where $\langle\cdot, \cdot\rangle$ denotes the usual scalar product. In other words, $X^{*}(x)=-N_{X}(x)$, where $N_{X}(x)$ is the normal cone of $X$ at $X$ (see [12]).

Given $x \in S$, it is well-known that, since $\gamma$ is a strictly convex norm, saying that no $y \in S$ verifies $\gamma_{a}(y)<\gamma_{a}(x)$ for all $a \in A$ is equivalent to saying that no $y \in S$ verifies $\left(\gamma_{a}(y) \leqslant \gamma_{a}(x) \forall a \in A\right.$, with some inequality strict) $[10,13]$, thus the concepts of efficiency and weak efficiency coincide:

$\mathbf{W E}(\gamma, A, S)=\mathbf{E}(\gamma, A, S)$

In order to characterize $\mathbf{E}(\gamma, A, S)$, some properties of strictly convex norms are needed. These properties are stated in Lemmas 1-4: Lemma 1 is a consequence of a more general result given in [11], and the proof is not repeated here. Lemmas 2-4 are new, and the proofs can be found in the Appendix.

Lemma 1. For any $x \in S$, the following statements are equivalent:

(i) There exists no $y \in S$ such that $\gamma_{a}(y)<\gamma_{a}(x)$ for all $a \in A$.

(ii) $S^{*}(x) \cap \operatorname{conv}\left(\bigcup_{a \in A} \partial \gamma_{a}(x)\right) \neq \emptyset$.

Lemma 2. The following statements are equivalent:

(i) $0 \in \operatorname{conv}\left(\bigcup_{a \in A} \partial \gamma(a)\right)$,

(ii) $0 \in \operatorname{conv}(A)$,

(iii) $0 \in \bigcup_{a \in \operatorname{conv}(A)} \partial \gamma(a)$.

Recall that, if $x=0$, then $\partial \gamma(x)=B^{0}$, whilst, for $x \neq 0, \partial \gamma(x)$ is an exposed face of $B^{0}$, see e.g. [3].

Lemma 3. Let $a, b, c \in b d(B), u, v, w \in b d\left(B^{0}\right)$ be such that

$u \in \partial \gamma(a), \quad v \in \partial \gamma(b), \quad w \in \partial \gamma(c)$.

(i) If $a \neq \pm b$ and $c=\lambda a+\mu b$ for some $\lambda, \mu>0$, then there exist $\alpha, \beta>0$ such that $w=\alpha u+\beta v$.

(ii) If $a \neq-b$ and $w=\alpha u+\beta v$ for some $\alpha, \beta \geqslant 0$, then there exist $\lambda, \mu \geqslant 0$ such that $c=\lambda a+\mu b$.

Lemma 4. For any closed convex cone $C$ with vertex at 0 , the following statements are equivalent:

(i) $C \cap \operatorname{conv}\left(\bigcup_{a \in A} \partial \gamma(a)\right) \neq \emptyset$,

(ii) $C \cap\left(\bigcup_{a \in \operatorname{conv}(A)} \partial \gamma(a)\right) \neq \emptyset$.

Given a point $x \in \mathbb{R}^{2}$, denote by $\operatorname{proj}_{\gamma}, s(x)$ the point in $S$ closest to $x$ with respect to $\gamma$, i.e.,

$\operatorname{proj}_{;, S}(x)=\arg \min _{y \in S} \gamma_{x}(y)$.

Since $S$ is closed and convex and $\gamma$ is a strictly convex norm, $\operatorname{proj}_{, S}$ is always well-defined.

For any set $X \subset \mathbb{R}^{2}$, denote also by $\operatorname{proj}_{\gamma} s(X)$, the set

$\operatorname{proj}_{\gamma_{,} S}(X)=\left\{\operatorname{proj}_{\gamma, S}(x): x \in X\right\}$.

With this notation, we are in position to characterize the set $\mathbf{E}(\gamma, A, S)$ of efficient points for problem $\mathbf{P}(\gamma, A, S)$, showing that the characterization given in 
[1] for the euclidean norm remains valid for general strictly convex norms.

Theorem 1. Let $S$ be a nonempty closed convex set in $\mathbb{R}^{2}$, and let $\gamma$ be a strictly convex norm. Then, for any nonempty compact set $A \subset \mathbb{R}^{2}$,

$\mathbf{E}(\gamma, A, S)=\mathbf{W E}(\gamma, A, S)=\operatorname{proj}_{\gamma, S}(\operatorname{conv}(A))$

Proof. Let $x \in S$; by Lemma $1, x \in \mathbf{W E}(\gamma, A, S)$ $(=\mathbf{E}(\gamma, A, S))$ iff

$S^{*}(x) \cap \operatorname{conv}\left(\bigcup_{a \in A} \partial \gamma_{a}(x)\right) \neq \emptyset$.

As $\operatorname{conv}\left(\bigcup_{a \in A} \partial \gamma_{a}(x)\right)=\operatorname{conv}\left(\bigcup_{b \in x-A} \partial \gamma(b)\right), S^{*}(x)$ is a closed convex cone with vertex at 0 , and $x-A$ is compact, Lemma 4 applies, and we have

$x \in \mathbf{W E}(\gamma, A, S)$ iff $S^{*}(x) \cap\left(\bigcup_{b \in \operatorname{conv}(x-A)} \partial \gamma(b)\right) \neq \emptyset$.

In other words, $x \in \mathbf{W E}(\gamma, A, S)$ iff $\exists b \in \operatorname{conv}(x-A)=$ $x-\operatorname{conv}(A)$ such that $S^{*}(x) \cap \partial \gamma(b) \neq \emptyset$, which occurs iff $\exists a^{*} \in \operatorname{conv}(A)$ such that $S^{*}(x) \cap \partial \gamma_{a^{*}}(x) \neq \emptyset$. By Lemma 1 (with $A=\left\{a^{*}\right\}$ ), nonvoidness of $S^{*}(x) \cap$ $\partial \gamma_{a^{*}}(x)$ is equivalent to $x$ being equal to $\operatorname{proj}_{\gamma, S}\left(a^{*}\right)$. Hence, $x \in \mathbf{W E}(\gamma, A, S)$ iff $x \in \operatorname{proj}_{\gamma, S}(\operatorname{conv}(A))$.

Remark 1. As a consequence of Theorem 1 in [7], when $A$ is finite, WE $(\gamma, A, S)$ equals the set of Weber points, i.e., the optimal solutions to problems of the form

$\min _{x \in S} \sum_{a \in A} \lambda_{a} \gamma_{a}(x)$

when $\lambda=\left(\lambda_{a}\right)_{a \in A}$ varies in the set of nonnegative nonzero vectors.

Hence, Theorem 1 implies that the set of Weber points for constrained problems equals the closestpoint projection (with respect to $\gamma$ ) of the set of Weber points without constraints, a result more precise than those given in [5].

Remark 2. The proof of Theorem 1 (in fact, the technical precedent lemmas) heavily relies on the fact that $\gamma$ is a norm, thus its ball $B$ is symmetric with respect to the origin. Extensions of Theorem 1 to general strictly convex gauges with asymmetric balls, seem to require different tools than those used in this paper. See also the recent paper [8] for a different approach to the problem.

\section{Acknowledgements}

The research of the first author has been partially financed by the Dirección General de Investigación Cientifica y Técnica, Spain, under grant PB93-0927.

\section{Appendix}

Proof of Lemma 2 (part (i) $\Leftrightarrow$ (ii)). Since $\gamma$ is a norm, it follows that $\partial \gamma(-a)=-\partial \gamma(a)$ for all $a \in \mathbb{R}^{2}$. Hence,

$0 \in \operatorname{conv}\left(\bigcup_{a \in A} \partial \gamma(a)\right)$ iff $0 \in \operatorname{conv}\left(\bigcup_{a \in A} \partial \gamma(-a)\right)$

By Lemma 1 as $\left(\mathbb{R}^{2}\right)^{*}(x)=\{0\}$, one has

$$
\begin{aligned}
& 0 \in \operatorname{conv}\left(\bigcup_{a \in A} \partial \gamma(-a)\right) \\
& \quad \text { iff }\left(\mathbb{R}^{2}\right)^{*}(0) \cap \operatorname{conv}\left(\bigcup_{a \in A} \partial \gamma_{a}(0)\right) \neq \emptyset,
\end{aligned}
$$

iff there exists no $y \in \mathbb{R}^{2}$ such that $\gamma_{a}(y)<\gamma_{a}(0)$ $\forall a \in A$, which is equivalent to $0 \in \mathbf{W E}\left(\gamma, A, \mathbb{R}^{2}\right)$. Hence,

$0 \in \operatorname{conv}\left(\bigcup_{a \in A} \partial \gamma(a)\right)$ iff $0 \in \mathbf{W E}\left(\gamma, A, \mathbb{R}^{2}\right)$.

By Corollary 1 of [11],

$0 \in \mathbf{W E}\left(\gamma, A, \mathbb{R}^{2}\right)$ iff $0 \in \mathbf{W E}\left(\gamma, A^{\prime}, \mathbb{R}^{2}\right)$ for some

finite $A^{\prime} \subset A$

Since $0 \in \mathbf{W E}\left(\gamma, A^{\prime}, \mathbb{R}^{2}\right)=\operatorname{conv}\left(A^{\prime}\right)$ for all finite $A^{\prime}$ (see [13]), and $\operatorname{conv}(A)=\bigcup\left\{\operatorname{conv}\left(A^{\prime}\right): A^{\prime} \subset A, A^{\prime}\right.$ is finite $\}$, it follows that $0 \in \operatorname{conv}\left(\bigcup_{a \in A} \partial \gamma(a)\right)$ iff $0 \in \operatorname{conv}(A)$, as asserted.

Proof of Lemma 2 (part (ii) $\Leftrightarrow$ (iii)). $0 \in \operatorname{conv}(A)$ iff $\exists a^{*} \in \operatorname{conv}(A)$ such that 0 minimizes in $\mathbb{R}^{2}$ the function $\gamma_{a^{*}}$. As $\gamma_{a^{*}}$ is convex, 0 minimizes $\gamma_{a^{*}}$ iff $0 \in \partial \gamma\left(-a^{*}\right)=-\partial \gamma\left(a^{*}\right)$. Hence, $0 \in \operatorname{conv}(A)$ iff $0 \in$ $\bigcup_{a \in \operatorname{conv}(A)} \partial \gamma(a)$, as asserted.

Proof of Lemma 3. We only prove part (i); part (ii) can be proven with similar arguments. 
As $u \in \partial \gamma(a), v \in \partial \gamma(b), \pm a, \pm b \in b d(B)$, and $B$ is symmetric with respect to 0 , one has:

(1) $\langle a, u\rangle=1$,

(2) $\langle b, v\rangle=1$,

(3) $|\langle x, u\rangle| \leqslant 1$ and $|\langle x, v\rangle| \leqslant 1$ for all $x \in B$.

In particular, $|\langle a, v\rangle| \leqslant 1$ and $|\langle b, u\rangle| \leqslant 1$. Furthermore, these inequalities are strict; indeed, if $\langle a, v\rangle=1$ (respect. $\langle a, v\rangle=-1$ ), the line $\langle v, x\rangle=1$ would support $B$ at $a$ and $b$ (resp. $-a$ and $b$ ), implying, because of the convexity of $B$, that the whole segment with extreme points $a$ and $b$ (resp. $-a$ and $b$ ) is contained in $b d(B)$, contradicting the assumption that $\gamma$ is a strictly convex norm. Hence, one has:

(4) $|\langle a, v\rangle|<1$,

(5) $|\langle b, u\rangle|<1$.

As, by assumption, $c=\lambda a+\mu b \in B,(1)-(2)$ imply (by (3), for $x=c$ ):

(6) $|\lambda+\mu\langle b, u\rangle| \leqslant 1$,

(7) $|\lambda\langle a, v\rangle+\mu| \leqslant 1$.

The vectors $u$ and $v$ are linearly independent; indeed, otherwise, as $\gamma^{0}(u)=\gamma^{0}(v)=1$, we would have that $u= \pm v$, contradicting (1)-(4). Hence, $\{u, v\}$ is a basis in $\mathbb{R}^{2}$, thus there exist $\alpha, \beta \in \mathbb{R}$ such that $w=\alpha u+\beta r$.

Observe that $w \in b d\left(B^{0}\right)$; hence, the line $\langle w, x\rangle=1$ supports $B$, thus

(8) $|\alpha\langle b, u\rangle+\beta| \leqslant 1$,

(9) $|x+\beta\langle a, v\rangle| \leqslant 1$.

Furthermore, as $w \in \partial \gamma(c)$ and $\langle w, c\rangle=1$, we also have:

(10) $\alpha\{\lambda+\mu\langle b, u\rangle\}+\beta\{\lambda\langle a, v\rangle+\mu\}=1$.

Define the segment $\Gamma$ in $\mathbb{R}^{2}$,

$\Gamma=\left\{(\bar{\alpha}, \bar{\beta}) \in \mathbb{R}^{2}:(\bar{\alpha}, \bar{\beta})\right.$ verifies $(8),(9)$ and $\left.(10)\right\}$

which, of course, contains the point $(\alpha, \beta)$.

In order to show that $\alpha$ and $\beta$ are strictly positive, we first show that $\Gamma$ is included in the nonnegative quadrant $\Gamma^{\prime}=\left\{(\bar{\alpha}, \bar{\beta}) \in \mathbb{R}^{2}: \bar{\alpha} \geqslant 0, \bar{\beta} \geqslant 0\right\}$, by showing that the vertices of $\Gamma$ are in $\Gamma^{\prime}$. The vertices of $\Gamma$ are among the points obtained by replacing one of the inequalities in (8) or (9) by an equality.

Let us study separately the different cases:

Case 1: $\bar{\alpha}\langle b, u\rangle+\bar{\beta}=1$

It leads to the values:

$\bar{x}=(1-\mu-\lambda\langle a, v\rangle) /(\lambda(1-\langle a, v\rangle\langle b, u\rangle))$,

$\bar{\beta}=(\lambda-(1-\mu)\langle b, u\rangle) /(\lambda(1-\langle a, v\rangle\langle b, u\rangle))$.
By (4), (5) and (7), it immediately follows that $\bar{\alpha} \geqslant 0$. On the other hand, (9) implies that $\lambda \geqslant|1-\mu|$; indeed, by (9),

$$
\begin{aligned}
1 \geqslant & |\bar{\alpha}+\bar{\beta}\langle a, v\rangle| \\
= & \mid((1-\mu-\lambda\langle a, v\rangle) \\
& +\langle a, v\rangle(\lambda-(1-\mu)\langle b, u\rangle)) /(\lambda(1-\langle a, v\rangle\langle b, u\rangle)) \mid \\
= & |(1-\mu)(1-\langle a, v\rangle\langle b, u\rangle) /(\lambda(1-\langle a, v\rangle\langle b, u\rangle))| \\
= & |(1-\mu) / \lambda|, \text { thus }|1-\mu| \leqslant|\lambda|=\lambda,
\end{aligned}
$$

as asserted.

Hence, by (5),

$\lambda-(1-\mu)\langle b, u\rangle \geqslant \lambda-|1-\mu| \geqslant 0$.

As $i .>0,(4)$ and (5) imply that $\lambda(1-\langle a, v\rangle$ $(b, u\rangle)>0$; hence, $\bar{\beta} \geqslant 0$.

Case $2: \bar{\alpha}\langle b, u\rangle+\bar{\beta}=-1$

It leads to the values

$\bar{x}=(1+\mu+\lambda\langle a, v\rangle) / \lambda(1-\langle a, v\rangle\langle b, u\rangle)$,

$\vec{\beta}=-(\lambda+(1+\mu)\langle b, u\rangle) / \lambda(1-\langle a, v\rangle\langle b, u\rangle)$.

First, (5) and (6) imply that

$1 \geqslant \lambda+\mu\langle b, u\rangle \geqslant \lambda-\mu|\langle b, u\rangle|>\lambda-\mu$,

thus $\lambda<1+\mu$. On the other hand, $(\bar{\alpha}, \bar{\beta})$ must verify (9), which is readily seen to be equivalent to $\lambda \geqslant \mid 1+$ $\mu \mid=1+\mu$, what is a contradiction.

Hence, the solution of $(10)$ and $\bar{\alpha}\langle b, u\rangle+\bar{\beta}=-1$ does not give a feasible point.

Due to the symmetry in $\bar{\alpha}, \bar{\beta}, \lambda, \mu$ in $\Gamma$ and constraints (1)-(7), similar results are obtained for the cases

$\bar{\alpha}+\bar{\beta}\langle a, v\rangle= \pm 1$.

Hence, all the extreme points of $\Gamma$ are contained in $\Gamma^{\prime}$, thus $\Gamma \subset \Gamma^{\prime}$; as $(\alpha, \beta) \in \Gamma$, it follows that $\alpha, \beta \geqslant 0$.

Furthermore, $\alpha$ and $\beta$ are both strictly positive; otherwise, $w=\alpha u$ for some $\alpha>0$ or $w=\beta v$ for some $\beta>0$; suppose that $w=\alpha u$ for some $\alpha>0$; as $1=\gamma^{0}(w)=\gamma^{0}(u)$, we would have that $w=u$. Hence, the line $\langle u, x\rangle=1$ would support $B$ both at $a$ and $c$, thus the nontrivial segment with extreme points $a$ and $c$ would be contained in $b d(B)$, which is a contradiction (recall that $\gamma$ is a strictly convex norm). 
With this we have shown part (i).

Proof of Lemma 4 (part (i) $\Rightarrow$ (ii)). As $0 \in C$, the result follows from Lemma 2 if $0 \in$ conv $\left(\bigcup_{a \in A} \partial \gamma(a)\right)$. Hence, we further assume that $0 \notin \operatorname{conv}\left(\bigcup_{a \in A} \partial \gamma(a)\right)$ (or, equivalently, $0 \notin \operatorname{conv}(A)$ ).

Consider the planar convex cone $A$,

$\Lambda=\left\{u \in \mathbb{R}^{2}: u=\lambda \xi\right.$ for some

$$
\left.\xi \in \operatorname{conv}\left(\bigcup_{a \in A} \partial \gamma(a)\right), \lambda \geqslant 0\right\}
$$

$A$ is a planar closed convex cone, and $A \neq \mathbb{R}^{2}$; indeed, it is easily seen that $\Lambda$ is a convex cone; closedness follows from the fact that $\operatorname{conv}\left(\bigcup_{a \in A} \partial \gamma(a)\right)$ is compact (see, e.g. [11]); as, by assumption, $0 \notin \operatorname{conv}\left(\bigcup_{a \in A} \partial \gamma(a)\right)$ and conv $\left(\bigcup_{a \in A} \partial \gamma(a)\right)$ is compact and convex, it follows that $\Lambda \neq \mathbb{R}^{2}$. Furthermore, it is straightforward to check that the extreme rays of $A$ are necessarily elements of $\bigcup_{a \in A} \partial \gamma(a)$, i.e., there exist $a_{1}, a_{2} \in A, \xi_{1} \in \partial \gamma\left(a_{1}\right), \xi_{2} \in \partial \gamma\left(a_{2}\right)$ such that

$\Lambda=\left\{u \in \mathbb{R}^{2}: u=t_{1} \xi_{1}+t_{2} \xi_{2}\right.$

for some $\left.t_{1}, t_{2} \geqslant 0\right\}$.

Furthermore, $\left(1 / \gamma\left(a_{1}\right)\right) a_{1} \neq-\left(1 / \gamma\left(a_{2}\right)\right) a_{2}$ because, otherwise, $0 \in \operatorname{conv}(A)$.

Let $d \in C \cap \operatorname{conv}\left(\bigcup_{a \in A} \partial \gamma(a)\right)$. As $d \in A$, and $d \neq 0$, it follows that

$d=\lambda_{1} \xi_{1}+\lambda_{2} \xi_{2}$ for some $\lambda_{1}, \lambda_{2} \geqslant 0, \lambda_{1}+\lambda_{2}>0$

Let $d^{\prime}=d / \gamma^{0}(d)$. One has:

- $d^{\prime}=\alpha \xi_{1}+\beta \xi_{2}$ for some $\alpha, \beta \geqslant 0, \alpha+\beta>0$.

- There exists $c \in b d(B)$ such that $d^{\prime} \in \partial \gamma(c)$ (because $\left.d^{\prime} \in b d\left(B^{0}\right)\right)$.

As $\xi_{i} \in \partial \gamma\left(a_{i} / \gamma\left(a_{i}\right)\right),(i=1,2), a_{1} / \gamma\left(a_{1}\right) \neq-a_{2} /$ $\gamma\left(a_{2}\right)$, and $a_{i} / \gamma\left(a_{i}\right) \in b d(B),(i=1,2)$, by Lemma 3 (part ii),

$c=\lambda a_{1}+\mu a_{2}$ for some $\lambda, \mu \geqslant 0, \lambda+\mu>0$

Let $c^{\prime}$ be the vector $c^{\prime}=c /(\lambda+\mu) \in \operatorname{conv}(A)$.

It follows that $d^{\prime} \in \partial \gamma\left(c^{\prime}\right) \subset \bigcup_{a \in \operatorname{conv}(A)} \partial \gamma(a)$, and $d^{\prime} \in C$.

Hence, $\bigcup_{a \in \operatorname{conv}(A)} \partial \gamma(a) \cap C \neq \emptyset$, as asserted.

Proof of Lemma 4 (part (ii) $\Rightarrow$ (i)). The result follows from Lemma 2 if $0 \in \bigcup_{a \in \operatorname{conv}(A)} \partial \gamma(a)$. Hence, we further assume that $0 \notin \bigcup_{a \in \operatorname{conv}(A)} \partial \gamma(a)$, i.e., $0 \notin$ $\operatorname{conv}(A)$. Consider the planar convex cone $\Gamma$,

$\Gamma=\left\{u \in \mathbb{R}^{2}: u=\lambda a\right.$ for some $\left.\lambda>0, a \in \operatorname{conv}(A)\right\}$.

Then, there exist $a_{1}, a_{2} \in A$ such that

$\Gamma=\left\{u: u=t_{1} a_{1}+t_{2} a_{2}\right.$ for some $t_{1}, t_{2} \geqslant 0$,

$$
\left.t_{1}+t_{2}>0\right\} \text {. }
$$

Furthermore, $\left(1 / \gamma\left(a_{1}\right)\right) a_{1} \neq-\left(1 / \gamma\left(a_{2}\right)\right) a_{2}$ (else, $0 \in$ $\operatorname{conv}(A)$ ).

Let $d \neq 0, d \in C \cap \bigcup_{a \in \operatorname{conv}(A)} \partial \gamma(a)$. There exists $a^{*} \in \operatorname{conv}(A)$ such that $d \in \partial \gamma\left(a^{*}\right)$. Furthermore, as $0 \notin \operatorname{conv}(A)$ and $a^{*} \neq 0$, it follows that $d \in b d\left(B^{0}\right)$.

As $\operatorname{conv}(A) \subset \Gamma$, there exist $\lambda, \mu \geqslant 0, \lambda+\mu>0$ such that

$a^{*}=\lambda a_{1}+\mu a_{2}$.

If $\left(1 / \gamma\left(a^{*}\right)\right) a^{*}=\left(1 / \gamma\left(a_{i}\right)\right) a_{i}$ for some $i=1,2$, $\left(1 / \gamma\left(a^{*}\right)\right) a^{*}=\left(1 / \gamma\left(a_{1}\right)\right) a_{1}$, say, the result holds because $d \in C$ and

$$
\begin{aligned}
d & \in \partial \gamma\left(a^{*}\right)=\partial \gamma\left(a^{*} / \gamma\left(a^{*}\right)\right)=\partial \gamma\left(a_{1} / \gamma\left(a_{1}\right)\right) \\
& =\partial \gamma\left(a_{1}\right) \subset \operatorname{conv}\left(\bigcup_{a \in A} \partial \gamma(a)\right)
\end{aligned}
$$

If it is not the case, we have that $\lambda>0, \mu>0$, and $\left(1 / \gamma\left(a_{1}\right)\right) a_{1} \neq\left(1 / \gamma\left(a_{2}\right)\right) a_{2}$.

Let $\xi_{1} \in \partial \gamma\left(a_{1}\right), \xi_{2} \in \partial \gamma\left(a_{2}\right)$. By Lemma 3, part (i), there exist $\alpha, \beta>0$ such that $d^{\prime}=\alpha \xi_{1}+\beta \xi_{2}$. Hence, the vector $d^{\prime \prime}=d^{\prime} /(\alpha+\beta)$ verifies:

- $d^{\prime \prime} \in C$,

- $d^{\prime \prime} \in \operatorname{conv}\left(\partial \gamma\left(a_{1}\right) \cup \partial \gamma\left(a_{2}\right)\right) \subset \operatorname{conv}\left(\bigcup_{a \in A} \partial \gamma(a)\right)$, thus (i) holds, as asserted.

\section{References}

[1] E. Carrizosa, E. Conde, F.R. Fernández and J. Puerto, "Efficiency in euclidean constrained location problems", Oper. Res. Lett. 14 (1993) 291-295.

[2] L.G. Chalmet, R.L. Francis and A. Kolen, "Finding efficient solutions for rectilinear distance location problems efficiently", Eur. J. Oper. Res. 6 (1981) 117-124.

[3] R. Durier and C. Michelot, "Geometrical properties of the Fermat-Weber problem", Eur. J. Oper. Res. 20 (1985) 332343.

[4] R. Durier and C. Michelot, "Sets of efficient points in a normed space", J. Math. Anal. Appl. 117 (1986) 506-528. 
[5] P. Hansen, D. Peeters and J.F. Thisse, "An algorithm for a constrained Weber problem", Management Sci. 28 (1982) 1285-1295

[6] P. Hansen, J. Perreur and J.F. Thisse, "Location Theory, dominance and convexity: Some further results", Oper. Res. 28 (1980) 1241-1250.

[7] T.J. Lowe, J.F. Thisse, J.E. Ward and R.E. Wendell, "On efficient solutions to multiple objective mathematical programs", Management Sci. 30 (1984) 1346-1349.

[8] M. Ndiaye, "Efficiency in constrained continuous location", Working paper no. 8/1995 of the Laboratoire d'Analyse Appliquée et Optimisation, Université de Bourgogne.

[9] F. Plastria, Continuous location problems and cutting plane algorithms, Ph.D. Dissertation, Vrije Universiteit Brussel, 1983.
[10] F. Plastria, "Points efficaces en localisation continue", Cahiers de Centre d'Etude en Recherche Operationelle de l'ULB (CCERO) 25 (1983), 329-332.

[11] F. Plastria and E. Carrizosa, "A geometrical characterization of weakly efficient points", to appear in: J. Optim. Theory Appl. (1996).

[12] R.T. Rockafellar, Convex Analysis, Princeton University Press, Princeton, NJ, 1970.

[13] J.F. Thisse, J.E. Ward and R.E. Wendell, "Some properties of Location problems with block and round norms", Oper. Res. 32 (1984) 1309-1327.

[14] R.E. Wendell and A.P. Hurter, "Location Theory, dominance and convexity", Oper. Res. 21 (1973) 314-320.

[15] R.E. Wendell, A.P. Hurter and T.J. Lowe, "Efficient points in location problems", AIIE Trans. 9 (1977) 238-246. 